\title{
Search for Beyond SM Higgs Bosons
}

\author{
S. Tsuno* on behalf of the ATLAS and CMS Collaborations \\ High Energy Accelerator Research Organization, KEK, \\ 1-1 Oho, Tsukuba, Ibaraki 305-0801, Japan \\ *E-mail: Soshi.Tsuno@cern.ch
}

\begin{abstract}
This article summarizes the latest results within the context of searches for Higgs boson production and properties beyond the Standard Model obtained with ATLAS and CMS experiments in summer 2017.
\end{abstract}

Keywords: Higgs; LHC; ATLAS; CMS.

\section{Introduction}

Recent studies of the Higgs boson ${ }^{1,2}$ with the ATLAS $^{3}$ and CMS $^{4}$ experiments suggest the existence of a spontaneous symmetry breaking phenomena in the electroweak sector. The observation of couplings of Higgs boson to fermions ${ }^{5,6}$ indeed proved the existence of the mass generation mechanism through the scalar Higgs field. However, the nature of this mechanism has not yet been precisely established. Several underlying questions like the hierarchy problem, unification of the forces, mystery of the asymmetry of the matter and anti-matter in universe, dark matter and the origin of the dark energy in universe are not explained by the Standard Model (SM). To answer such fundamental questions a new theory, beyond the SM, is required. The quest to discover new particles beyond those predicted by the SM is therefore a primary task of the LHC experiments in addition to performing precise measurements of the Higgs boson.

This article summarizes the latest results within the context of the searches for additional Higgs bosons, beyond that predicted by the SM, presented by the ATLAS and CMS experiments in summer 2017. An overview of the searches for MSSM Higgs bosons is discussed in Sec. 2, a summary of the use of the Higgs boson as a tool in searches for new physics is presented in Sec. 3, searches for anomalous Higgs couplings and rare decays are presented in Sec. 4, and the summary is given in Sec. 5.

\section{Searches for MSSM Higgs bosons}

\subsection{Overview}

The Minimal Supersymmetric Standard Model (MSSM) ${ }^{7-9}$ is an extension of the SM inducing the supersymmetric partners of the SM particles. The MSSM requires two Higgs doublets with opposite hypercharge which results in one $\mathrm{CP}$-odd $(A)$, two CP-even $(h, H)$ neutral Higgs bosons and two charged Higgs bosons $\left(H^{ \pm}\right)$with 
two additional mixing parameters, $\alpha$ the mixing angle of $h$ and $H$, and $\tan \beta$ the ratio of the vacuum expectation values of the up-type and down-type fermions in the two Higgs doublets.

Extensive searches for these Higgs bosons have been carried out over the years. For example, Fig. 1 summarizes the 95\% CL exclusion contours ${ }^{10}$ in the MSSM $m_{h}^{\bmod +}$ scenario $^{11}$ (with $\mu=+200 \mathrm{GeV}$ ), obtained from selected CMS analyses that have been performed with the LHC Run 1 dataset. The $m_{h}^{\bmod +}$ scenario is chosen to be the mass of the lightest CP-even Higgs boson, $m_{h}$, is close to the measured mass of the Higgs boson that was discovered at the LHC. The colored filled areas correspond to the excluded regions in $m_{A}$ and $\tan \beta$. In the Fig. 1 on the right in addition to the direct exclusion contours, the constraint that is obtained from the compatibility of the scenario with the couplings of the SM Higgs boson when interpreted as the $h$ is also displayed.

The couplings of the Higgs bosons to down-type fermions are enhanced for large $\tan \beta$, which results in a large production rate for the Higgs bosons with couplings to $\tau$ leptons and $b$-quarks. Therefore, direct searches for the heavy $H / A$ bosons decaying to $\tau$ leptons ${ }^{12,13}$ and $b$-quarks ${ }^{14}$ are appropriate approaches to high $\tan \beta$ region, while searches for Higgs bosons coupled to the up-type fermions explore the low $\tan \beta$ region. Since almost all Higgs boson measurements so far indicate the obtained parameters are consistent with the $\mathrm{SM}$, that is, low $\tan \beta$ region is preferred, the number of analyses searching in the low $\tan \beta$ region has rapidly grown during Run 2 of the LHC. The charged Higgs boson $H^{ \pm}$with couplings to the $t$ - and $b$-quark ${ }^{15,16}$ is one channel which can explore the low $\tan \beta$ region. Searches for the decays of a heavy Higgs boson, $A$, to a light Higgs boson, $h$, and the SM weak boson $Z^{17,18}$ also provides indirect constraints. Analyses may also search for interference effects in the top-pair production process caused by the decay $H \rightarrow t \bar{t}$ in high mass region ${ }^{19}$. Finally, searches for charged Higgs bosons decaying to $\tau \nu^{16,20}$ provide global constraints ranging from the low to high $m_{A}$ regions.

In the following, only the selected physics analyses, $H / A \rightarrow \tau \tau$ and $H^{ \pm} \rightarrow \tau \nu / t b$, which represent the most powerful channels in constraining the MSSM parameter space of $\tan \beta$ as a function of Higgs mass, are presented as benchmark searches.

\subsection{Search for $H / A \rightarrow \tau \tau$}

The analysis ${ }^{12}$ is performed with $36.1 \mathrm{fb}^{-1}$ data at a centre-of-mass energy of $13 \mathrm{TeV}$ collected with the ATLAS detector in 2015 and 2016. Figure 2 shows the typical lowest order Feynman diagrams for gluon-gluon fusion production (a), $b$-quark associated production with 4 flavor scheme (b) and 5 flavor scheme (c) of a neutral MSSM Higgs boson. The $b$-quark associated production mode is enhanced in the high $\tan \beta$ region. The search is therefore split according to the presence or absence of jets originating from $b$-quarks in the final state as well as the decay mode of the 


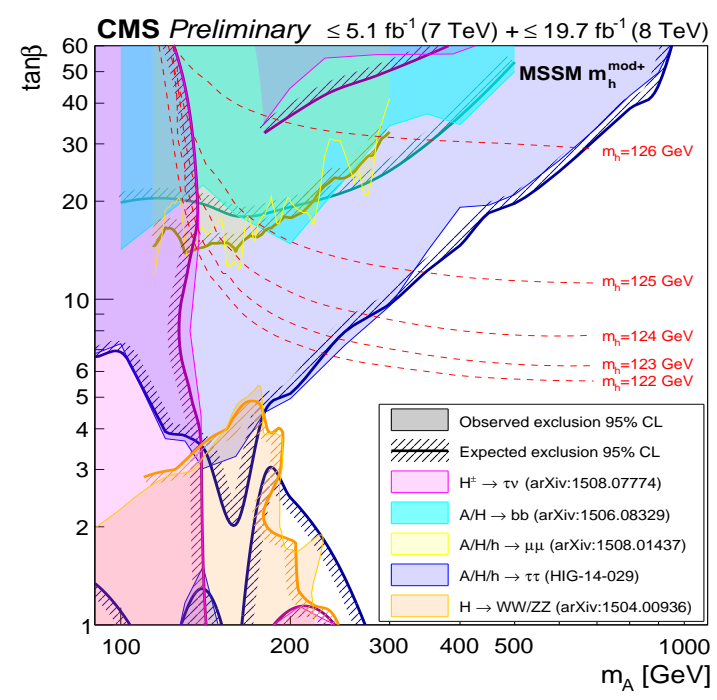

Fig. 1. 95\% CL exclusion contours, in the MSSM $m_{h}^{\bmod +}$ scenario (with $\mu=+200 \mathrm{GeV}$ ), which is obtained by selected CMS analyses that have been performed with the LHC Run 1 dataset. The colored filled areas correspond to the excluded regions in $m_{A}$ and $\tan \beta$. The colored (slightly darker shaded) lines with indicated hatches to the regions that were expected to be excluded, based on the null-hypothesis assumption of a SM-like Higgs sector. In the figure on the right in addition to the direct exclusion contours, the constraint is also displayed that is obtained from the compatibility of the scenario with the couplings of the SM Higgs boson when interpreted as the $h$.

$\tau$ leptons. The final discriminant is $m_{\mathrm{T}}^{\text {tot }}$, defined as

$$
m_{\mathrm{T}}^{\text {tot }}=\sqrt{\left(p_{\mathrm{T}}^{\tau_{1}}+p_{\mathrm{T}}^{\tau_{2}}+E_{\mathrm{T}}^{\text {miss }}\right)^{2}-\left(\vec{p}_{\mathrm{T}}^{\tau_{1}}+\vec{p}_{\mathrm{T}}^{\tau_{2}}+\vec{E}_{\mathrm{T}}^{\text {miss }}\right)^{2}}
$$

where $p_{\mathrm{T}}^{\tau_{1 / 2}}$ is the transverse momentum of the visible component of the $\tau$ decay products, $E_{\mathrm{T}}^{\mathrm{miss}}$ is the opposite direction of the transverse vector sum of all detected particles from the collision events, which represents the direction in transverse plane of the sum of the momenta of all neutrinos produced in $\tau$ decays. The dominant backgrounds are $W+$ jets, $t \bar{t}$ and multi-jet processes where a jet is mis-identified as a hadronically decaying $\tau$-lepton. They are estimated from data. The dominant experimental uncertainty is associated with the modeling of high- $p_{\mathrm{T}}$ hadronic $\tau$ decays which cannot be verified in data due to the lack of a sufficiently large control sample of $Z \rightarrow \tau \tau$ events $^{21}$. To address this, hadronic $\tau$ identification is studied in a sample of high- $p_{\mathrm{T}}$ di-jet events, where the modeling of the detector response to such events is assumed to be the same as for high- $p_{\mathrm{T}}$ hadronic $\tau$ events. An additional uncertainty of $\sim 20-25 \%$ is assigned for high- $p_{\mathrm{T}}$ hadronic $\tau$ events.

Figure 3 presents the $m_{\mathrm{T}}^{\text {tot }}$ distribution in the $b$-tagged category (a) and the observed and expected 95\% CL upper limits on $\tan \beta$ as a function of $m_{A}$ in the MSSM $m_{h}^{\bmod +}$ scenario (b). Comparing with Fig. 1, the exclusion limit is largely expanded in Run 2, mostly owing to the increased production cross section from 
$8 \mathrm{TeV}$ to $13 \mathrm{TeV}$. The region $\tan \beta>25$ at $m_{A}=1 \mathrm{TeV}$ is excluded. Model independent limits of $\sigma \times \operatorname{Br}(\phi \rightarrow \tau \tau)=0.0058-0.85 \mathrm{pb}$ for gluon-gluon fusion and $0.0041-0.95 \mathrm{pb}$ for $b$-quark associated processes in the mass range of $0.2-2.25 \mathrm{TeV}$ are also obtained.

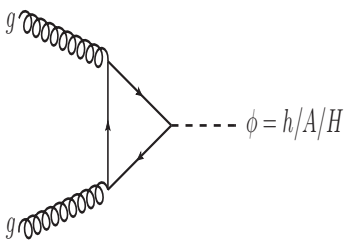

(a) gluon-gluon fusion

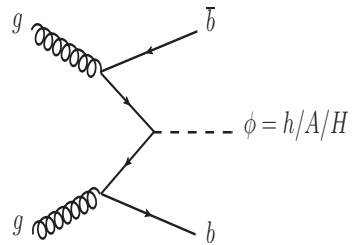

(b) 4FS associated production

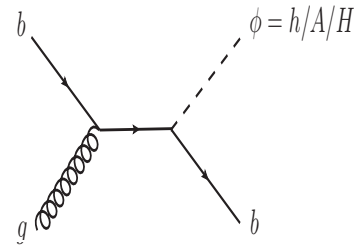

(c) 5FS associated production

Fig. 2. Lowest order Feynman diagrams for gluon-gluon fusion production (a), $b$-quark associated production with 4 flavor scheme (b) and 5 flavor scheme (c) of a neutral MSSM Higgs boson.

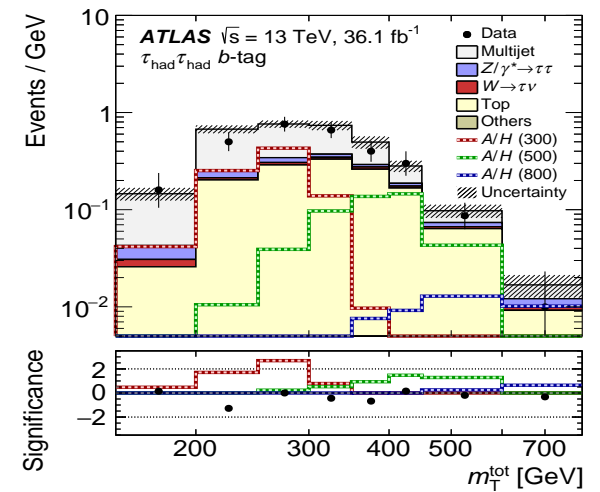

(a) $m_{\mathrm{T}}^{\text {tot }}$

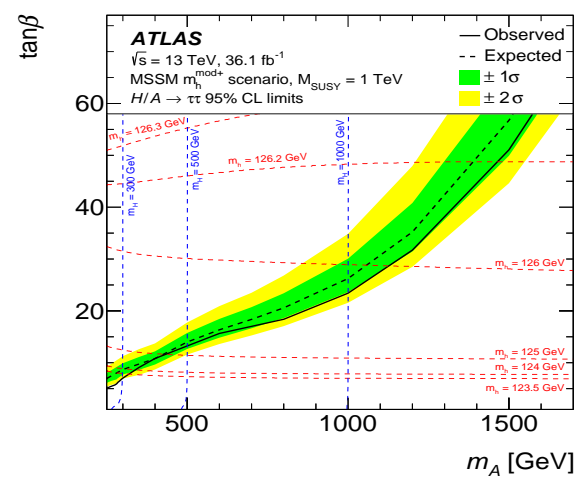

(b) $95 \%$ CL upper limit

Fig. 3. (a) $m_{\mathrm{T}}^{\text {tot }}$ for the $b$-tag category in the hadronic $\tau$ channel. The hypothetical Higgs boson signals with masses of 300,500 and $800 \mathrm{GeV}$ and $\tan \beta=10$ are also overlaid. (b) The observed and expected $95 \%$ CL upper limits on $\tan \beta$ as a function of $m_{A}$ in the MSSM $m_{h}^{\bmod +}$ scenario. Dashed lines of constant $m_{h}$ and $m_{H}$ are shown in red and blue, respectively.

\subsection{Search for $H^{ \pm} \rightarrow \tau \nu$ and $t b$}

The main production mode of the charged Higgs boson at high mass $\left(m_{H^{ \pm}}>\right.$ $\left.m_{\text {top }}(=172.5 \mathrm{GeV})\right)$ is through the associated production of $t b H$, as shown in Fig. 4. The $H^{ \pm}$predominantly decays into $\tau \nu$ or $t b$ final state depending on $m_{H^{ \pm}}$and $\tan \beta$. In both case, the $t$ - and $b$-quarks in the final state are associated with the $H^{ \pm}$, so that the analyses ${ }^{15,16,20}$ require at least one $b$-quark jet and high jet 
multiplicity. The dominant background is from the $t \bar{t}+$ jets process as well as a jet faking the hadronic $\tau$-lepton from $W$ +jets and multi-jet events. For fully hadronic events without containing $e$ or $\mu$ in the final state, the $E_{\mathrm{T}}^{\text {miss }}$ trigger is used in combination with the hadronic $\tau$ trigger. The final discriminant is the distribution of the transverse mass between hadronic $\tau$ and missing transverse energy for the $H \rightarrow \tau \nu$ mode,

$$
m_{\mathrm{T}}=\sqrt{2 \cdot p_{\mathrm{T}}^{\tau}\left|E_{\mathrm{T}}^{\mathrm{miss}}\right|\left(1-\cos \Delta \phi\left(\vec{p}_{\mathrm{T}}^{\tau}, \vec{E}_{\mathrm{T}}^{\mathrm{miss}}\right)\right) .}
$$

On the other hand, for the $H \rightarrow t b$ decay mode, the reconstruction of the invariant mass of the final state is non-trivial and the analysis relies instead on a fit to a multivariate discriminant. The dominant experimental uncertainties are the jet energy scale uncertainty and $E_{\mathrm{T}}^{\mathrm{miss}}$ as well as the modeling of the hadronic $\tau$ events. For the multivariate analysis, the theory uncertainty is large, at around $50 \%$, due to the uncertainty in estimating the number of additional jets produced in $t \bar{t}$ events. Figure 5 shows the observed and expected 95\% CL upper limits on $\tan \beta$ as a function of $m_{H^{ \pm}}$of the $H^{ \pm} \rightarrow \tau \nu$ (a) and $H^{ \pm} \rightarrow t b$ (b) analyses, respectively. The $H^{ \pm} \rightarrow \tau \nu$ analysis is sensitive to the high $\tan \beta$ region while the $H^{ \pm} \rightarrow t b$ is sensitive to the low $\tan \beta$ region. These analyses extend the constraints on the MSSM parameter space using Run 2 data.

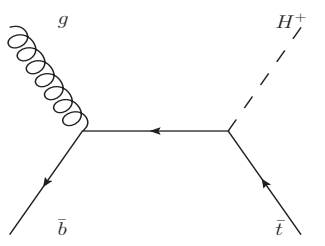

(a) 5FS associate production

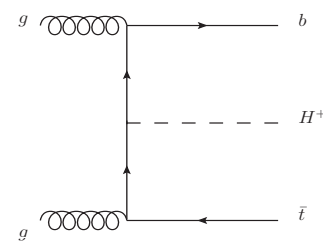

(b) 4FS associated production

Fig. 4. Lowest order Feynman diagrams for associated production with 4 flavor scheme (a) and 5 flavor scheme (b) of a charged MSSM Higgs boson.

\section{The Higgs boson as a probe of new physics}

\subsection{Overview}

The observation of the Higgs boson ${ }^{22,23}$ opens up the possibility of its use as a tool to probe new physics. Many theories beyond the SM predict new particles decaying into Higgs bosons. If such a particle has a mass larger than that of the Higgs boson $(125 \mathrm{GeV})$, the decay products can become highly boosted leading a collimated shower of final state particles. The SM Higgs boson decays into a $b$-quark pair around $60 \%$ of the time. In this case, the two $b$-quark jets are reconstructed as a merged single jet object with a mass close to the Higgs boson mass. Other decay 


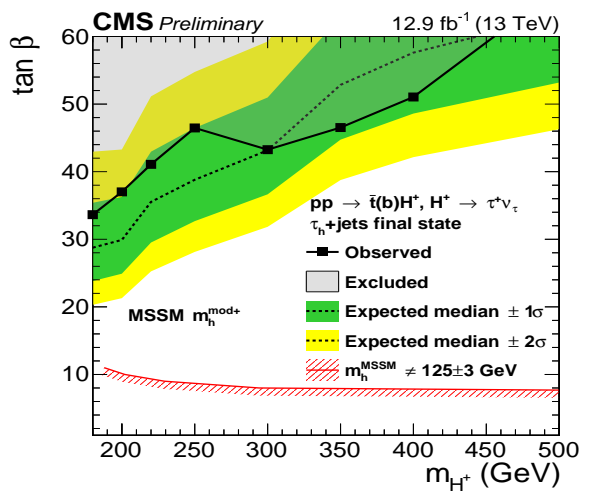

(a) $H^{ \pm} \rightarrow \tau \nu$

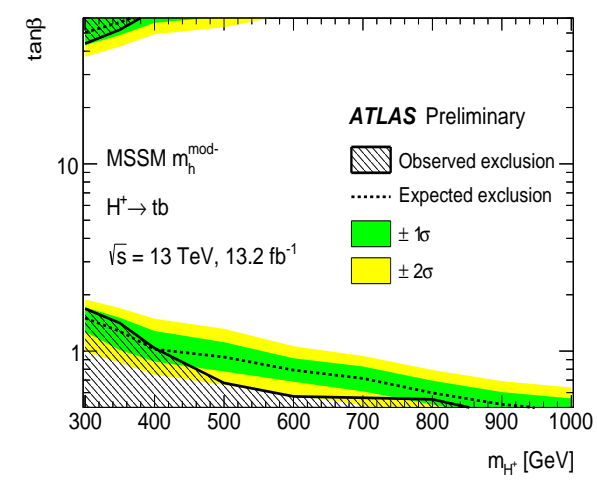

(b) $H^{ \pm} \rightarrow t b$

Fig. 5. The observed and expected 95\% CL upper limits on $\tan \beta$ as a function of $m_{H} \pm$ of the $H^{ \pm} \rightarrow \tau \nu(\mathrm{a})$ and $H^{ \pm} \rightarrow t b(\mathrm{~b})$ analyses, respectively.

modes can be considered in addition to the $b$-quark pair decay. Therefore, such analyses are typically split into two categories, a merged category and a resolved category. The merged category targets events where the decay products, typically jets, are reconstructed as a single object and exploit the sub-structure within the merged object to maximize the sensitivity. The resolved category attempts to resolve two distinct objects identified with, for example, the standard $b$-quark jet or hadronic $\tau$ reconstruction algorithms. This categorization depends on the mass of the new particle and typically $m_{X}=1 \mathrm{TeV}$ is the boundary between the merged and resolved categories. Many analyses search for a heavy resonance decaying into bosons (including the Higgs boson). In the following sections, only the decay of heavy resonances into final states including a Higgs boson are considered.

\subsection{Search for $X \rightarrow V h$}

A new particle $X$ can decay into a Higgs boson, $h$, and SM $W$ or $Z$ boson, where the $h$ is required to decay to a $b$-quark pair and the $W$ or $Z$ bosons are required to decay leptonically or hadronically into $\ell \ell, \ell \nu, \nu \nu, j j^{24}$. The resonance mass $m_{V h}$ is well-reconstructed from the 4 -vectors from the measured objects in both the merged and resolved categories. When a neutrino is in the final state, the $m_{\mathrm{T}}^{\text {tot }}$ variable is used instead. Figure 6 shows the $m_{V h}$ distribution in the resolved (a) and merged (b) categories for a particular analysis channel with leptons and $b$-quark jets in the final state. The signal events with $m_{X}=1.5 \mathrm{TeV}$ are also overlaid as 10 times of the predicted cross section for a benchmark model. The resolution for the $m_{\mathrm{T}}^{\text {tot }}$ is about $30-40 \%$ at $m_{X}=1.5 \mathrm{TeV}$.

The $95 \%$ CL upper limits on the production cross section of the $A$ multiplied by its branching ratio into the $Z h$ final state and the branching ratio of $h \rightarrow b \bar{b}$ are obtained as a function of resonance mass in Fig. 7 for gluon-fusion (a) and $b$-quark 
associated (b) production processes, respectively. The data are also interpreted in terms of limits at $95 \% \mathrm{CL}$ on the $2 \mathrm{HDM}$ parameters of $\tan \beta$ and $\cos (\beta-\alpha)$ for several benchmark models.

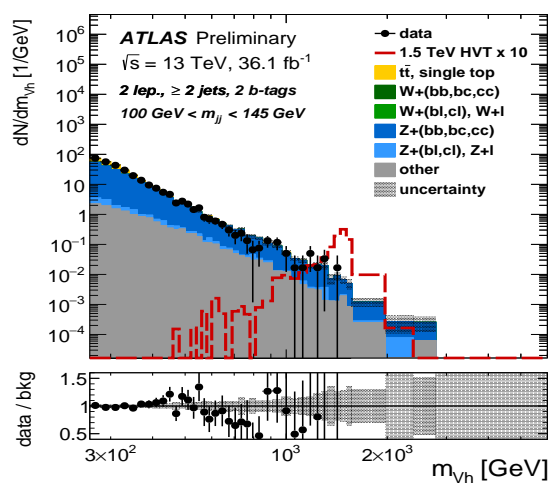

(a) Resolved category

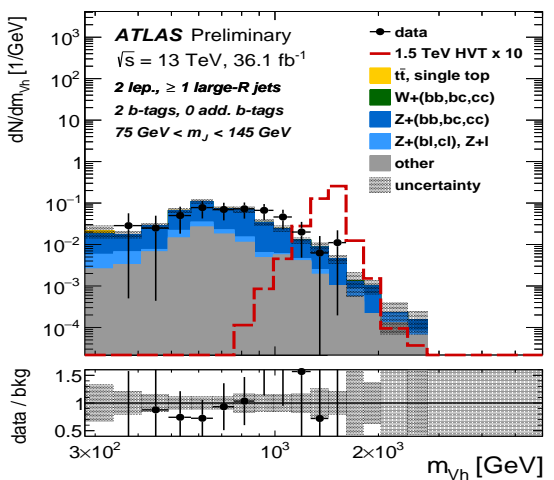

(b) Merged category

Fig. 6. The resonance mass $m_{V h}$ in the resolved (a) and merged (b) categories for a particular analysis channel with leptons and $b$-quark jets in the final state. The signal events with $m_{X}=1.5 \mathrm{TeV}$ are also overlaid as 10 times of the predicted cross section for a benchmark model.

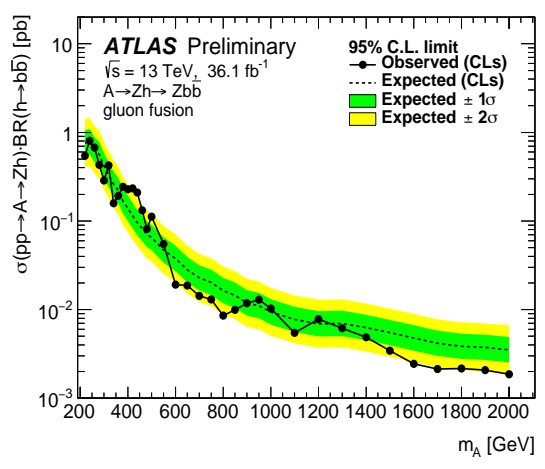

(a) gluon-fusion production

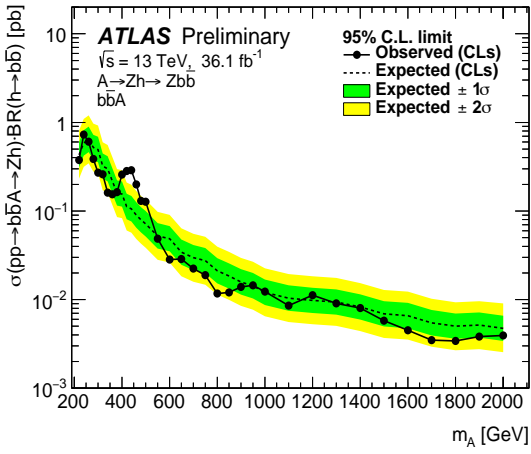

(b) b-quark associated production

Fig. 7. Upper limits at the $95 \% \mathrm{CL}$ on the product of the production cross-section for $p p \rightarrow A$ and the branching ratios for $A \rightarrow Z h$ and $h \rightarrow b \bar{b}$. The possible signal components of the data are interpreted assuming (a) pure gluon-fusion production, and (b) pure $b$-quark associated production. 


\subsection{Search for $X \rightarrow h h$}

Given the unique topology of the $h$ decay, backgrounds can be well suppressed when a new resonance decays into $h h$. The considered decay modes are $b \bar{b} b \bar{b}^{25,26}, b \bar{b} \ell \nu \ell \nu^{27}$, $b \bar{b} \tau \tau^{28}$, and $b \bar{b} \gamma \gamma^{29}$. Figure 8 (a) shows the di-jet invariant mass distribution $m_{j j}^{\text {red }}$ in the $b \bar{b} b \bar{b}$ channel, where the invariant mass of two small-jets inside the merged object (jet) is required to be $105<m_{j j}^{\text {small }}<135 \mathrm{GeV}$, the soft component inside jets is trimmed and selection of the N-subjettiness ${ }^{25}$ is required. The signal cross section is assumed to be $20 \mathrm{pb}$ for all the mass hypotheses at 1400, 1800 and $2500 \mathrm{GeV}$ for illustration purposes. Only a very small background contribution is present in the large resonance mass region $\left(m_{X}>1.5 \mathrm{TeV}\right)$. Figure $8(\mathrm{~b})$ presents the observed and expected 95\% CL upper limits on the product of cross section and the branching ratio $\sigma(g g \rightarrow X) \times B(X \rightarrow h h)$ obtained by different analyses assuming spin-0 hypothesis in an extended mass range beyond $1 \mathrm{TeV}$.

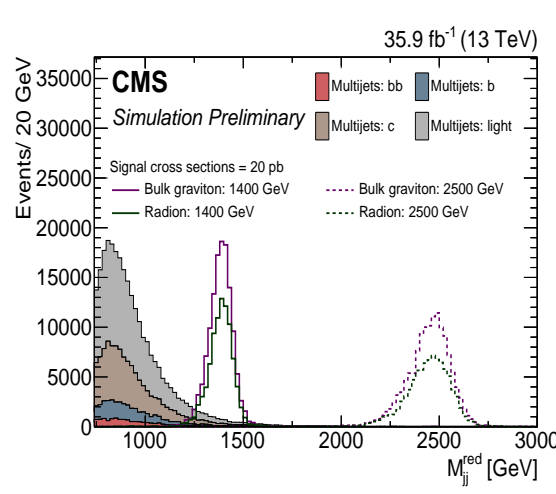

(a) reduced dijet invariant mass, $m_{j j}^{\text {red }}$

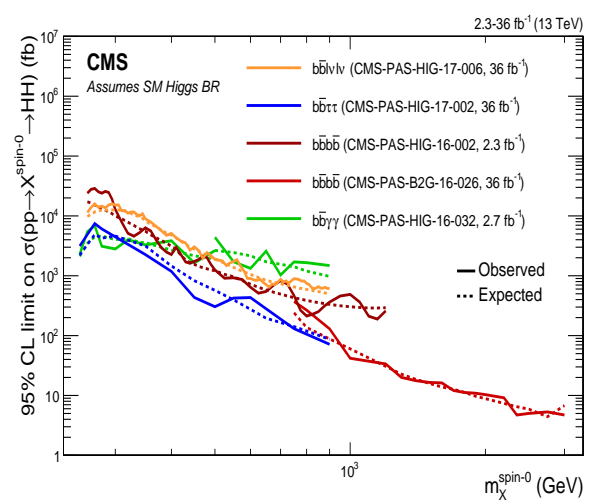

(b) $95 \%$ CL upper limits

Fig. 8. (a) The reduced di-jet invariant mass $m_{j j}^{\text {red }}$ in $b \bar{b} b \bar{b}$ channel, where the invariant mass of two small-jets inside the merged object (jet) is required to be $105<m_{j j}^{\text {small }}<135 \mathrm{GeV}$. The signal cross section is assumed to be $20 \mathrm{pb}$ for all the mass hypotheses at 1400, 1800 and $2500 \mathrm{GeV}$. (b) Observed and expected 95\% CL upper limits on the product of cross section and the branching ratio $\sigma(g g \rightarrow X) \times B(X \rightarrow h h)$ obtained by different analyses assuming spin-0 hypothesis in an extended mass range beyond $1 \mathrm{TeV}$.

\section{Anomalous Higgs couplings and rare decays}

\subsection{Overview}

Unlike the case of the SM gauge bosons, the fermion couplings in the Higgs sector is not universal across fermion flavors. Although the Yukawa couplings are predicted to be proportional to the mass of the fermion, the detailed structure of the Yukawa couplings has not yet been determined. This inspires the possibility of flavor violating decays through off-diagonal elements of the Yukawa mass matrix or rare decays 
involving new particles beyond the SM.

In this section, searches for rare and exotic decays of the Higgs boson are discussed.

\subsection{Search for flavor-violating decay modes}

The flavor-changing neutral current process is strictly forbidden in the framework of the SM, however searches for such processes in the decay of the Higgs boson represent important tests of flavor violation in the Higgs sector. High energy colliders provide many opportunities to study the Yukawa couplings of the third generation fermions. A search for lepton flavor violating decays of the $125 \mathrm{GeV}$ Higgs boson in the $\mu \tau$ and $e \tau$ decay mode, $H \rightarrow \mu \tau, e \tau$, has been carried out ${ }^{30,31}$. No significant excess over the SM background expectation is observed. The observed (expected) upper limits on the branching ratio of the Higgs boson are set to be $\operatorname{Br}(H \rightarrow \mu \tau)$ $<0.25(0.25) \%$ and $\operatorname{Br}(H \rightarrow e \tau)<0.61(0.37) \%$ at $95 \%$ CL. Constraints on the flavor violating Yukawa couplings, $\left|y_{\mu \tau}\right|,\left|y_{\tau \mu}\right|$, are obtained from these results as shown in Fig. 9 (a) for $\mu \tau$ mode. The flavor diagonal Yukawa couplings are approximated by their SM values. The green (yellow) band indicates the range that is expected to contain $68 \%$ (95\%) of all observed limit excursions from the expected limit. The shaded regions are constraints derived from null searches for $\tau \rightarrow 3 \mu$ (dark green) and $\tau \rightarrow \mu \gamma$ (lighter green). The purple diagonal line is the theoretical naturalness limit $y_{i j} y_{j i} \leq m_{i} m_{j} / v^{2}$, where $v$ is the vacuum expectation value $246 \mathrm{GeV}$. Upper limits on the off-diagonal $\mu \tau$ and $e \tau$ Yukawa couplings are derived as $\sqrt{\left|y_{\mu \tau}\right|^{2}+\left|y_{\tau \mu}\right|^{2}}<1.43 \times 10^{-3}$ and $\sqrt{\left|y_{e \tau}\right|^{2}+\left|y_{\tau e}\right|^{2}}<2.26 \times 10^{-3}$ at $95 \% \mathrm{CL}$, respectively.

Flavor violation in the quark sector is also tested using the $t$-quark decay, $t \rightarrow$ $q H^{32}$, where $q$ is an up-type quark, $c$ or $u$. The $H \rightarrow \gamma \gamma$ decay mode is used since it offers optimal sensitivity. The SM prediction is $\operatorname{Br}_{\mathrm{SM}}(t \rightarrow q H)=3 \times 10^{-15}$. Figure 9 (b) shows the invariant mass distribution of the two selected photons and a jet from the decay of the $t$-quark. The signal distributions are normalized assuming a branching ratio of $5 \%$. The limit is obtained to be $\operatorname{Br}(t \rightarrow q H)<2.4 \times 10^{-3}$ at $95 \%$ CL.

\subsection{Search for exclusive decay mode}

The Higgs boson can also decay into hadrons exclusively through loop diagrams as illustrated in Fig. 10. According to the SM, the branching ratios for the exclusive decays of the Higgs boson to a meson and photon, $J / \psi \gamma, \phi \gamma$ and $\rho \gamma$, are $2.95 \times 10^{-6}$, $2.31 \times 10^{-6}$, and $1.68 \times 10^{-7}$, respectively ${ }^{33}$. The mesons are experimentally identified using the decay modes of $J / \psi \rightarrow \mu^{+} \mu^{-}, \phi \rightarrow K^{+} K^{-}$and $\rho \rightarrow \pi^{+} \pi^{-}$.

Figure 11 shows the invariant masses $m_{K^{+} K^{-}}$(a) and $m_{K^{+} K^{-}}$(b) for the selected $\phi \gamma$ candidates ${ }^{34}$. The reconstructed mass resolution of $m_{K^{+} K^{-}}$is about 4 $\mathrm{MeV}$. The $m_{K^{+} K^{-}}$distribution exhibits a clear phi mass peak over the combinatoric 


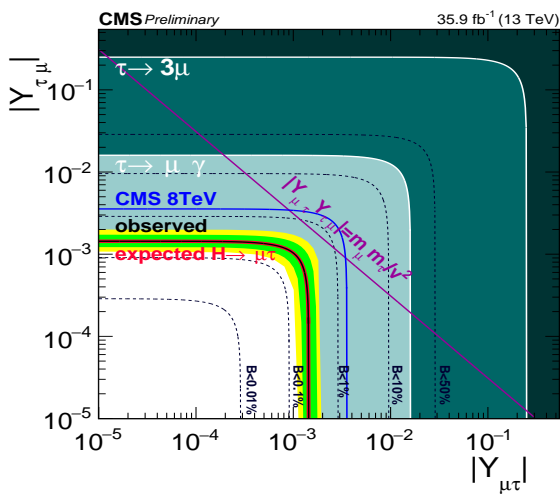

(a) Constraints of $\left|y_{\mu \tau}\right|$ and $y_{\tau \mu} \mid$

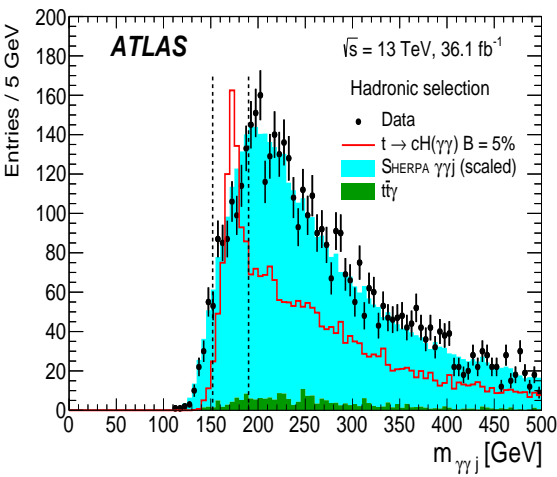

(b) Invariant mass, $m_{\gamma \gamma j}$

Fig. 9. (a) Constraints on the flavor violating Yukawa couplings, $\left|y_{\mu \tau}\right|, y_{\tau \mu} \mid$. The expected (red solid line) and observed (black solid line) limits are derived from the limit on $\operatorname{Br}(H \rightarrow \mu \tau)$. The flavor diagonal Yukawa couplings are approximated by their SM values. The green (yellow) band indicates the range that is expected to contain $68 \%(95 \%)$ of all observed limit excursions from the expected limit. The shaded regions are derived constraints from null searches for $\tau \rightarrow 3 \mu$ (dark green) and $\tau \rightarrow \mu \gamma$ (lighter green). The purple diagonal line is the theoretical naturalness limit $y_{i j} y_{j i} \leq m_{i} m_{j} / v^{2}$. (b) Invariant mass distribution of the two selected photons and a jet from $t$-quark. The signal distributions are normalized assuming a branching ratio of $5 \%$.

background. Combining with the $\gamma$, the Higgs (and $Z$ ) boson mass is reconstructed with high precision. No significant excess of events is observed above the background, in agreement with the SM expectations. Upper limits at 95\% CL on the branching ratios of the exclusive radiative Higgs boson decays to mesons are set as $\operatorname{Br}(H \rightarrow \phi \gamma)<4.8 \times 10^{-4}$ and $\operatorname{Br}(H \rightarrow \rho \gamma)<8.8 \times 10^{-4}$, respectively. The current limits on the branching fractions for such decays are two orders of magnitude higher than the SM prediction. However, assuming a dataset of $3000 \mathrm{fb}^{-1}$ at $\sqrt{s}=14 \mathrm{TeV}$, a limit of $\operatorname{Br}(H \rightarrow J / \psi \gamma)=5.5 \times 10^{-7}$ is expected $^{35}$.

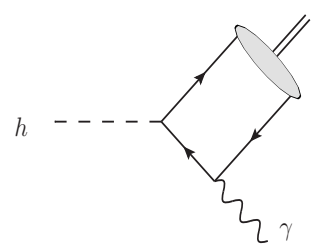

(a) Direct contribution

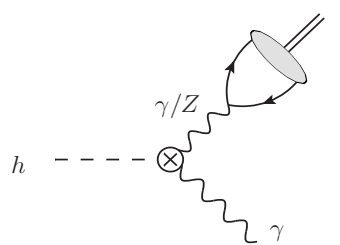

(b) Indirect contribution

Fig. 10. Feynman diagrams for direct (a) and indirect (b) contributions in the exclusive decay of $H \rightarrow V \gamma$. The crossed circle denotes the off-shell $H \rightarrow \gamma \gamma^{*}$ and $H \rightarrow \gamma Z^{*}$ amplitudes, which in the SM arise first at one-loop order. 


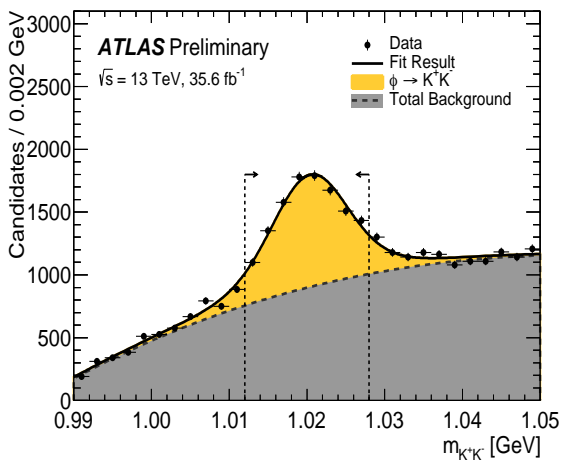

(a) $m_{K^{+} K^{-}}$

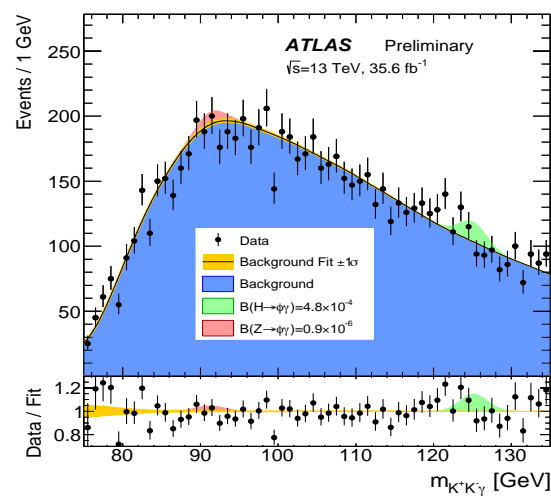

(b) $m_{K^{+} K^{-}}$

Fig. 11. The $m_{K^{+} K^{-}}$(a) and $m_{K^{+} K^{-} \gamma}$ (b) distributions for the selected $\phi \gamma$ candidates. The Higgs and $Z$ boson contributions for the branching ratio values corresponding to the observed 95\% CL upper limits are also shown.

\subsection{Search for exotic decay mode}

Several theories beyond the SM predict the existence of a dark sector that is weakly coupled with the SM particles. Due to the weakness of the coupling, the particles of the dark sector can have non-negligible lifetime which could decay beyond the beam-pipe or even outside the detector. The CMS experiment have searched for long lived particles which could decay inside the tracking detector volume $(c \tau \leq 100$ $\mathrm{mm})^{36}$, while the ATLAS experiment searches for decays within the region between the beam-pipe and the muon detectors $(3.8 \leq c \tau \leq 600 \mathrm{~mm})^{37}$. Limits on models predicting Higgs boson decays to neutral long-lived particles (dark photons $\gamma_{D}$ ) are derived as a function of the decay length, $c \tau_{\gamma_{D}}$ as shown in Fig. 12.

\section{Summary and prospects}

In this article, three different topics are discussed within the context of the latest searches for Higgs boson production and decays beyond that predicted in the SM.

Searches for MSSM Higgs bosons explore both the low and high $\tan \beta$ regions in term of the mass of $H / A$ boson. With current dataset of $36 \mathrm{fb}^{-1}$, an MSSM Higgs bosons are excluded up to $1 \mathrm{TeV}$ at $\tan \beta \sim 20$ with $m_{h}^{\bmod +}$ scenario. The HL-LHC will be sensitive to the region up to $m_{H / A}=400 \mathrm{GeV}$ for the whole parameter space, but the region around $\tan \beta \sim 10$ at high mass region is not excluded, and may require a future linear or circular collider to investigate.

The Higgs boson can be used as a tool to probe a new physics, where a new heavy particle may decay into Higgs bosons. When the mass of a particle is sufficiently larger than that of the $125 \mathrm{GeV}$ Higgs boson, its decay products can be largely boosted and reconstructed as single object. By resolving the individual decay products or reconstructing them as a merged object, the good rejection of background 


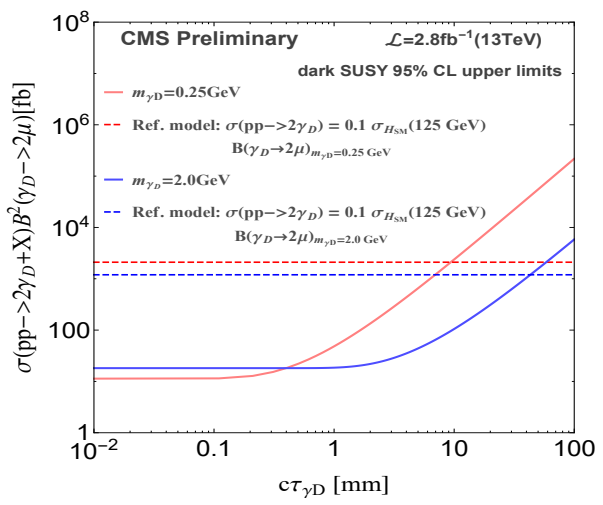

(a) CMS search

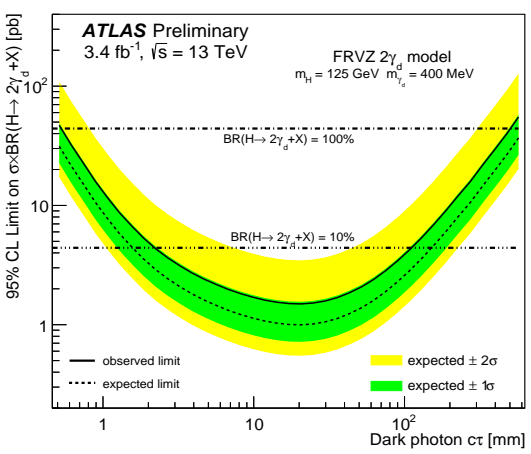

(b) ATLAS search

Fig. 12. The 95\% CL upper Limit on $\sigma\left(p p \rightarrow 2 \gamma_{D}+X\right) \times B r^{2}\left(\gamma_{D} \rightarrow \mu \mu\right)$ as a function of $c \tau_{\gamma_{D}}$ for two dark photon masses of 0.25 and $2.0 \mathrm{GeV}$ in CMS (b), and $0.4 \mathrm{GeV}$ in ATLAS (a).

processes can be achieved, leading to almost model independent exclusion limits which extend up to $1 \mathrm{TeV}$.

The search for anomalous Higgs couplings and rare decays also represent important tests of the nature of the Higgs boson, since the detailed structure of the Yukawa coupling is not yet well established. Searches for flavor violating Higgs boson decays have been performed by the ATLAS and CMS experiments and already lead to very stringent limits. The exclusive radiative decays of the Higgs boson to light mesons are also searched for. To date, the limits on these decays are far from the SM prediction, but in HL-LHC era, such decays may offer the possibility to study the Yukawa couplings of the first and second generation quarks. Searches for exotic Higgs boson decay modes have also been performed, including decays to dark matter candidates. The results from the collider experiments are very complementary to the programme of underground direct detection experiments.

In conclusion, it should be emphasized that both the ATLAS and CMS experiments are very active in the area of Higgs boson studies, both in terms of production and coupling measurements and searches for phenomena beyond the SM.

\section{References}

1. ATLAS and CMS Collaborations, Measurements of the Higgs boson production and decay rates and constraints on its couplings from a combined ATLAS and CMS analysis of the LHC pp collision data at $\sqrt{s}=7$ and 8 TeV, JHEP 08, p. 045 (2016).

2. CMS Collaboration, Precise determination of the mass of the Higgs boson and tests of compatibility of its couplings with the standard model predictions using proton collisions at 7 and 8 TeV, Eur. Phys. J. C75, p. 212 (2015).

3. ATLAS Collaboration, The ATLAS Experiment at the CERN Large Hadron Collider, JINST 3, p. S08003 (2008).

4. CMS Collaboration, The CMS experiment at the CERN LHC, JINST 3, p. S08004 
(2008).

5. ATLAS Collaboration, Evidence for the Higgs-boson Yukawa coupling to tau leptons with the ATLAS detector, JHEP 04, p. 117 (2015).

6. CMS Collaboration, Evidence for the direct decay of the $125 \mathrm{GeV}$ Higgs boson to fermions, Nature Phys. 10, 557 (2014).

7. A. Djouadi, The Anatomy of electro-weak symmetry breaking. II. The Higgs bosons in the minimal supersymmetric model, Phys. Rept. 459, 1 (2008).

8. G. C. Branco, P. M. Ferreira, L. Lavoura, M. N. Rebelo, M. Sher and J. P. Silva, Theory and phenomenology of two-Higgs-doublet models, Phys. Rept. 516, 1 (2012).

9. P. Fayet, Supersymmetry and weak, electromagnetic and strong interactions, Physics Letters B 64, 159 (1976).

10. CMS Collaboration, Summary results of high mass BSM Higgs searches using CMS run-I data, CMS-PAS-HIG-16-007 (2016).

11. M. Carena, S. Heinemeyer, O. Stl, C. E. M. Wagner and G. Weiglein, MSSM Higgs Boson Searches at the LHC: Benchmark Scenarios after the Discovery of a Higgs-like Particle, Eur. Phys. J. C73, p. 2552 (2013).

12. ATLAS Collaboration, Search for additional heavy neutral Higgs and gauge bosons in the ditau final state produced in $36 \mathrm{fb}^{-1}$ of pp collisions at $\sqrt{\mathrm{s}}=13 \mathrm{TeV}$ with the ATLAS detector, arXiv:1709.07242 (2017).

13. CMS Collaboration, Search for neutral MSSM Higgs bosons decaying to a pair of tau leptons in pp collisions, JHEP 10, p. 160 (2014).

14. CMS Collaboration, Search for neutral MSSM Higgs bosons decaying into a pair of bottom quarks, JHEP 11, p. 071 (2015).

15. ATLAS Collaboration, Search for charged Higgs bosons in the $H^{ \pm} \rightarrow t b$ decay channel in pp collisions at $\sqrt{s}=8$ TeV using the ATLAS detector, JHEP 03, p. 127 (2016).

16. CMS Collaboration, Search for a charged Higgs boson in pp collisions at $\sqrt{s}=8 \mathrm{TeV}$, JHEP 11, p. 018 (2015).

17. ATLAS Collaboration, Search for a CP-odd Higgs boson decaying to $Z$ h in pp collisions at $\sqrt{s}=8$ TeV with the ATLAS detector, Phys. Lett. B744, 163 (2015).

18. CMS Collaboration, Searches for a heavy scalar boson $H$ decaying to a pair of 125 GeV Higgs bosons hh or for a heavy pseudoscalar boson A decaying to Zh, in the final states with $h \rightarrow \tau \tau$, Phys. Lett. B755, 217 (2016).

19. ATLAS Collaboration, Search for heavy Higgs bosons $A / H$ decaying to a top quark pair in pp collisions at $\sqrt{s}=8$ TeV with the ATLAS detector, arXiv:1707.06025 (2017).

20. ATLAS Collaboration, Search for charged Higgs bosons produced in association with a top quark and decaying via $H^{ \pm} \rightarrow \tau \nu$ using pp collision data recorded at $\sqrt{s}=13$ TeV by the ATLAS detector, Phys. Lett. B759, 555 (2016).

21. ATLAS Collaboration, Measurement of the tau lepton reconstruction and identification performance in the ATLAS experiment using pp collisions at $\sqrt{s}=13 \mathrm{TeV}$, ATLASCONF-2017-029 (2017).

22. ATLAS Collaboration, Observation of a new particle in the search for the Standard Model Higgs boson with the ATLAS detector at the LHC, Phys. Lett. B716, 1 (2012).

23. CMS Collaboration, Observation of a new boson at a mass of $125 \mathrm{GeV}$ with the CMS experiment at the LHC, Phys. Lett. B716, 30 (2012).

24. ATLAS Collaboration, Search for heavy resonances decaying to $a W$ or $Z$ boson and a Higgs boson in final states with leptons and b-jets in $36.1 \mathrm{fb}^{-1}$ of pp collision data at $\sqrt{s}=13$ TeV with the ATLAS detector, ATLAS-CONF-2017-055 (2017).

25. CMS Collaboration, Search for heavy resonances decaying to a pair of Higgs bosons in the four $b$ quark final state in proton-proton collisions at $\sqrt{s}=13 \mathrm{TeV}$, CMS-PASB2G-16-026 (2017). 
26. CMS Collaboration, Search for resonant pair production of Higgs bosons decaying to two bottom quark-antiquark pairs in proton-proton collisions at $13 \mathrm{TeV}$, CMS-PASHIG-16-002 (2016).

27. CMS Collaboration, Search for resonant and nonresonant Higgs boson pair production in the bblnulnu final state in proton-proton collisions at $\sqrt{s}=13 \mathrm{TeV}$, arXiv:1708.04188 (2017).

28. CMS Collaboration, Search for Higgs boson pair production in events with two bottom quarks and two tau leptons in proton-proton collisions at $\sqrt{s}=13 \mathrm{TeV}$, arXiv:1707.02909 (2017).

29. CMS Collaboration, Search for Higgs boson pair production in the final state containing two photons and two bottom quarks in proton-proton collisions at $\sqrt{s}=13 \mathrm{TeV}$, CMS-PAS-HIG-17-008 (2017).

30. CMS Collaboration, Search for lepton flavour violating decays of the Higgs boson to $\mu \tau$ and $e \tau$ in proton-proton collisions at $\sqrt{s}=13 \mathrm{TeV}$, CMS-PAS-HIG-17-001 (2017).

31. ATLAS Collaboration, Search for lepton-flavour-violating decays of the Higgs and $Z$ bosons with the ATLAS detector, Eur. Phys. J. C77, p. 70 (2017).

32. ATLAS Collaboration, Search for top quark decays $t \rightarrow q H$, with $H \rightarrow \gamma \gamma$, in $\sqrt{s}=13$ TeV pp collisions using the ATLAS detector, JHEP 10, p. 129 (2017).

33. D. de Florian et al., Handbook of LHC Higgs Cross Sections: 4. Deciphering the Nature of the Higgs Sector, arXiv:1610.07922 (2016).

34. ATLAS Collaboration, Search for exclusive Higgs and $Z$ boson decays to $\phi \gamma$ and $\rho \gamma$ with the ATLAS Detector, ATLAS-CONF-2017-057 (2017).

35. ATLAS Collaboration, Search for the Standard Model Higgs and Z Boson decays to $J / \psi \gamma$ : HL-LHC projections, ATL-PHYS-PUB-2015-043 (2015).

36. CMS Collaboration, A Search for Beyond Standard Model Light Bosons Decaying into Muon Pairs, CMS-PAS-HIG-16-035 (2016).

37. ATLAS Collaboration, Search for long-lived neutral particles decaying into displaced lepton jets in proton-proton collisions at $\sqrt{s}=13 \mathrm{TeV}$ with the ATLAS detector, ATLAS-CONF-2016-042 (2016). 